\title{
BMJ Open Competencies for collaboration between general practitioners and medical specialists: a qualitative study of the patient perspective
}

\author{
Marijn Janssen (D , ${ }^{1}$ Cornelia R M G Fluit, ${ }^{2}$ Margaretha H Sagasser, ${ }^{3}$ \\ Loes H J Kusters, ${ }^{4}$ Nynke D Scherpbier-de Haan, ${ }^{5}$ Jacqueline de Graaf ${ }^{1,2}$
}

To cite: Janssen M,

Fluit CRMG, Sagasser MH, et al. Competencies for collaboration between general practitioners and medical specialists: a qualitative study of the patient perspective. BMJ Open 2020;10:e037043. doi:10.1136/ bmjopen-2020-037043

- Prepublication history and additional material for this paper are available online. To view these files, please visit the journal online (http://dx.doi org/10.1136/bmjopen-2020037043).

Received 16 January 2020 Revised 16 April 2020 Accepted 18 May 2020

\section{Check for updates}

(c) Author(s) (or their employer(s)) 2020. Re-use permitted under CC BY-NC. No commercial re-use. See rights and permissions. Published by BMJ.

${ }^{1}$ Department of Internal Medicine, Radboudumc, Nijmegen, The Netherlands ${ }^{2}$ Radboudumc Health Academy, Radboudumc, Nijmegen, The Netherlands

${ }^{3}$ Network of GP Specialty Training Institute in The Netherlands, Utrecht, The Netherlands

${ }^{4}$ Dutch Training Programme for Specialists in Elderly Care, Utrecht, The Netherlands

${ }^{5}$ Primary and Community Care, Radboudumc, Nijmegen, The Netherlands

Correspondence to

Marijn Janssen;

marijn.janssen@radboudumc.nl

\section{ABSTRACT}

Objectives To explore the patient view of competencies essential for doctors to provide good collaboration at the primary-secondary care interface.

Design We used a qualitative research approach. Focus groups with patients were conducted to explore their opinions of doctors' competencies to provide good collaboration between primary and secondary care doctors. Transcripts were analysed using thematic analysis.

Setting Dutch primary-secondary care interface. Participants Sixteen participants took part in five focus groups. Patients treated in both primary and secondary care, defined as having a minimum of two contacts with their general practitioner and two contacts with a medical specialty in the last 6 months, were included. Psychiatric patients and children were excluded from this study. Results Three groups of competencies were identified: (1) relationship building, both with patients and with other doctors; (2) transparent collaborating: be able to provide clarity on the process of collaboration and on roles and responsibilities of those involved and (3) reflective practising: to be willing to acknowledge mistakes, give and receive feedback and act as a lifelong learner.

Conclusions This focus group study enhances our understanding of the patient perspective on doctors' collaborative competencies at the primary-secondary care interface. With this information, doctors can improve their collaborative skills to a level that would meet their patients' needs. Patients expect doctors to be able to build relationships and act as reflective practitioners. Including patients in the collaborative process by giving them a role that is appropriate to their abilities and by making collaboration more explicit could help to improve collaboration between general practitioners and medical specialists.

\section{INTRODUCTION}

With populations ageing and chronic diseases and multimorbidity increasing, changes in the provision and organisation of healthcare are required. While care is getting more complex due to growing knowledge and technical possibilities, the tendency is to provide care in a primary healthcare setting as much
Strengths and limitations of this study

- This focus group study provides a broad view of the patient perspective on collaborative competencies of general practitioners and medical specialists, a subject scarcely studied.

- Interaction, the advantage of a focus group design, was a key factor in capturing the participants' perspective on collaborative competencies.

- The number and heterogeneity of study participants within groups were limited, which could affect the transferability.

- As the researchers had previous experience working at the primary-secondary care interface, this may have influenced the findings.

as possible. ${ }^{1-3}$ To provide high-quality care in the primary healthcare setting, collaboration with secondary care is essential to exchange knowledge and experience of different diseases and their management. ${ }^{45}$ Furthermore, such exchange between primary and secondary care is essential for the process of shared decision-making both in primary and secondary care, one of the cornerstones of personalised healthcare nowadays.

Collaboration at the primary-secondary care interface is a broad concept. It is intertwined with continuity of care, that is, the delivery of services by different providers in a coherent, logical and timely fashion for the patients. ${ }^{6}$ Primary-secondary care collaboration takes place in several ways: on a daily basis, it occurs in the form of referral, (curb side) consultation and discharge; in the context of chronic disease management, it occurs in the development of care pathways. Poor collaboration can lead to patients having reduced confidence in care providers and increasing anxiety. ${ }^{7}$ Research on discharge processes shows that transitions are associated with a substantial number of medical errors. ${ }^{8}$ 
In collaborative processes in healthcare, organisational, systemic and (inter) personal factors play a role $\mathrm{e}^{9}$ and can be targets for improving collaboration. Research on collaboration at the primary-secondary care interface reveals several barriers that could be overcome by improving the behaviour of doctors. Patients mention barriers such as difficulty accessing the primary care provider, his/her lack of knowledge, problems with clinicians' attitudes and communication issues. ${ }^{10}$ Doctors mention barriers such as not knowing one another, insufficient knowledge of each other's work environment, unclear roles and responsibilities, poor communication, lack of mutual respect and specialists questioning the general practitioners' (GPs) expertise. ${ }^{11-14}$

To overcome these barriers, we need to improve our understanding of the knowledge, skills and attitudes (or competencies) that doctors need to provide better collaboration. Research addressing this question is scarce. With the definition of competencies, doctors can gain insight into their collaborative behaviour in daily practice. Furthermore, these competencies can be implemented as learning objectives at all stages of medical education. Patients' perspective on collaborative competencies could be very useful here: as they experience care at both primary and secondary levels, they can provide a more holistic perspective than most doctors who only work in either primary or secondary care. Research has shown, furthermore, that there are differences in the patients' and providers' perspectives of the continuity of care. ${ }^{6}$ Therefore, the aim of our study was to explore patients' views of competencies that are required for doctors to provide good collaboration at the primary-secondary care interface.

\section{METHODS}

\section{Context}

The study was carried out in the Netherlands, where GPs are the doctors providing primary care. General practices are situated at a mean distance of $1 \mathrm{~km}$ from their patients, and hospitals are located at a mean distance of 5 $\mathrm{km}$. Access to secondary care is only possible after referral by a primary care provider. Most medical specialists, referred to as specialists in the remainder of this article, work in hospitals, and some work in independent treatment day care centres or in specialist clinical centres. The patients' journey between primary and secondary care has been visualised in figure 1 .

\section{Study design}

We conducted a qualitative study using focus groups followed by thematic analysis to gain insight into the knowledge, skills and attitudes that contribute to collaboration between doctors in primary and secondary care. We used focus groups because we assumed that the interaction between the different participants could lead to more in-depth insights. ${ }^{15}$

Our research team consisted of six females: one GP (NDS-dH), one medical specialist $(\mathrm{JdG})$, one resident in medical specialty training and $\mathrm{PhD}$ student in the field of interprofessional collaboration (MJ), one educationalist and doctor (CRMGF), one educationalist (MHS) and one medical student (LHJK). All except LHJK had experience conducting qualitative research prior to this research. When conducting and analysing the focus groups, all researchers worked at the Radboud University Medical Center.

Figure 1 Journey of patients between primary and secondary care. Not every patient travels from 1 to 6 . Some patients remain with the general practitioner (GP) after phone or web consultation of the specialist by the GP (1), several patients go back to the GP after visiting the specialist (1-2-3-5), without hospitalisation. 


\section{Participants}

We wanted to explore the views of patients treated in both primary and secondary care. Patients were eligible if they had had a minimum of two contacts in person, by phone or by email with their GP and two contacts with the same specialty over the past 6 months. We excluded psychiatric patients because of the possible unwanted influence of their disease on focus group participation and vice versa. We focused our study on patients over the age of 18 . We aimed to include 6-10 participants in each focus group with a heterogeneous group composition to catch a variety of opinions. We hypothesised that the following characteristics could influence participants' opinions on collaboration and aimed to create heterogeneity in terms of: treating specialism, age, sex and educational level (low, medium or high according to the International Standard Classification of Education classification). ${ }^{16}$

To recruit participants, we contacted 13 regional GPs to approach patients meeting the inclusion criteria and provide interested patients with an information letter. Patients were contacted by telephone by the primary researcher. To ensure diversity of patients, we aimed to include patients from different hospital settings (university, regional) and different areas (urban, rural). To ensure inclusion of patients below the age of 40, who are treated less often in both primary and secondary care, we contacted Adolescents \& Young Adults (AYA) national, ${ }^{17}$ an organisation providing support to patients with cancer between the ages of 18 and 35. Besides a refund for travel expenses, participants received no compensation for participation.

\section{Data collection}

One interviewer (MJ) guided the focus groups. She did not know the interviewees beforehand. To enhance credibility of the results, an assistant researcher (LHJK, CRMGF or TW) joined the focus groups and took notes. Four focus groups took place at the Radboud University Medical Center and one at a location of the Netherlands Comprehensive Cancer Organisation (Integraal Kankercentrum Nederland). We used a semistructured interview guide with open-ended questions in all focus group meetings (online supplementary file 1). After an open question to capture the participants' first thoughts about their experiences of collaboration between GPs and medical specialists, subsequent questions focused on doctors' competencies required for such collaboration. ${ }^{15}$ At the end of each focus group, the interviewer summarised the discussion and allowed the participants to comment on the accuracy and validity of the information. After focus groups two, three and four, preliminary results were discussed by the research team. To gain deeper insight into specific findings, we added questions for subsequent focus groups. The focus group interviews were audio taped, transcribed verbatim and anonymised. Anonymised interview transcripts were returned to participants to give them insight into the data used for analysis. ${ }^{18}$ It was our intention to conduct focus groups until thematic saturation had been reached.

\section{Data analysis}

We used a thematic analysis approach, which is a qualitative method that identifies, interprets, analyses and defines themes derived from data. ${ }^{19}$ All transcripts were coded by two researchers, MJ and LHJK or MJ and CRMGF. We used an inductive approach to analysis. Each researcher independently identified meaningful units that provided information on what doctors should know, and what skills and attitudes they should show. Differences in codes were solved by discussion, and discussions in the research team led to the formation of themes that we organised into groups of competencies and subcompetencies. Data analysis started as soon as the first data had been gathered. In this way, data gathering and data analysis formed an iterative process, promoting dependability. $^{2021}$ During this analytical process, LHJK and MJ kept memos and used a reflexive journal to document coding and analysis. To stimulate reflexivity within the research team, researchers' viewpoints on the data and the analytic process were critically questioned and discussed. $^{21} 22$ Atlas.ti software V.7.1.4 was used to organise the data.

\section{Patient involvement}

Although this study focuses on the patient perspective, patients were not involved in the design of the study. AYA national helped to recruit young participants. Patients received transcripts of the focus groups and will receive a copy of the article once published. In this study, anonymity was guaranteed, and participation was voluntary and had no consequences for the patients' treatment. Before participating in the focus groups, participants had to sign a written informed consent form.

\section{RESULTS}

Sixteen patients from 13 different GPs working in 9 practices participated in 5 focus groups between May 2016 and December 2017. Patients were treated in 10 different hospitals, both university and regional ones. Several patients were treated at the research site. Out of 26 patients approached by the researcher, 2 did not want to participate. When we started planning focus groups, we experienced that creating heterogeneous groups as we had planned was not feasible due to patient availabilities. We decided to let availability prevail over heterogeneity. Due to time or health constraints, four patients had to withdraw their participation beforehand. Ten participants had to cancel at the last minute, and two of these were able to attend another focus group. These last-minute cancellations resulted in four focus groups with three participants. Thematic saturation was reached after four groups, but the perspective of patients under the age of 40 was lacking. As younger patients might have different experiences with and opinions about care and 


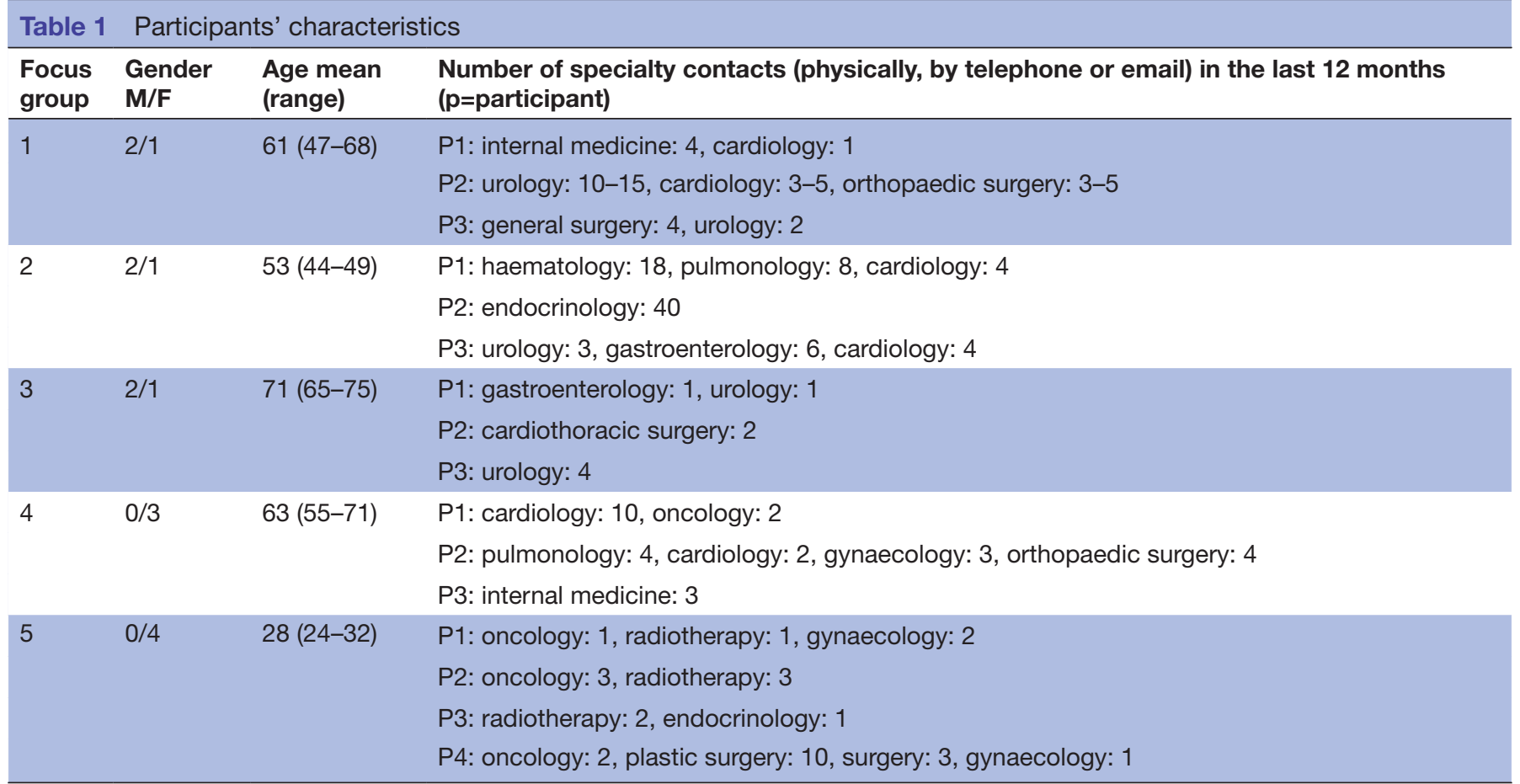

collaboration, we decided to plan an additional focus group with younger patients. As recruiting was difficult, this fifth group was conducted with only four participants. Analysis of the fifth focus group revealed no new themes in addition to the previous groups.

Participants' characteristics are shown in table 1.

We identified three groups of doctors' competencies: relationship building, transparent collaborating and reflective practising (table 2).

\section{Relationship building}

The ability to build relations with patients and colleague doctors was seen as an important competency. When asked about what doctors should do to realise this, all patients mentioned listening, both to each other and to the patient.

\section{Relationship with patients}

In all focus groups, patients valued their relationship with doctors in the collaborative process. If doctors saw them as a person and were able to connect with them, they assumed that they were probably good at connecting with other doctors as well. Some participants felt that having a good relationship with their doctor made it easier to address collaborative issues, leading to improvement of care.

If you have a good connection with your GP, you feel free to address issues, like 'I visited the specialist six months ago and you still do not have any information; I am unhappy about that.' And then we can discuss whether the GP or I will contact the hospital. (Patient 3 FG 4)
The patients took various roles in the collaborative process, by communicating between their GP and the specialist, for example, or by keeping a personal medical file. They liked to be seen and valued for the role they

\begin{tabular}{ll}
\hline Table 2 Competencies and subcompetencies \\
\hline Competencies & Subcompetencies \\
\hline Relationship & Relationship with patients \\
building & See the patient as a human being \\
& Recognise and acknowledge the role of \\
& the patient \\
& Relationship with doctors \\
& Have a collaborative attitude \\
& Provide good oral and written \\
& communication \\
& Have knowledge of the other person and \\
& work context \\
& Make collaboration visible \\
& Make sure patients know about or are able \\
& to follow information exchange \\
Transparent & Show respect for your colleague doctors \\
collaborating & towards the patient and acknowledge their \\
& role in caring for the patient
\end{tabular}

Make clear arrangements about roles and responsibilities in primary-secondary care collaboration and follow-up on them

$\begin{array}{ll}\text { Reflective } & \text { Acknowledge mistakes towards patients and } \\ \text { practising } & \text { each other and give and receive feedback to } \\ & \text { one another } \\ & \text { Lifelong learning; collaborate to stay up-to- } \\ & \text { date on diseases, diagnoses and treatments }\end{array}$


could play as experts in their own care process. However, they felt that this was not possible for every patient or in every situation. Participants suggested that doctors could discuss the desired level of patient participation with every patient at several moments in the collaborative process. Although patients wanted to be seen and valued for their role, they did not want to be made responsible for their medical files.

I think they should have all the medical facts in their files. Then you can add from your own perspective. Otherwise you feel responsible for having to give the correct medical facts. I found that hard. (Patient 4, FG 5)

\section{Relations between doctors}

Besides connecting with patients, doctors in primary and secondary care should build relations with each other. A collaborative attitude and some knowledge of their counterpart and his or her workplace is needed to do so. Participants expected that, with such knowledge, doctors would be better able to prepare and impart confidence to their patients for transitions between primary and secondary care and provide better continuity of care. One participant mentioned the following:

What's important is a culture in which you don't see yourself as an island, but in which everything is directly related to each other. ...It's like, you must all deal directly with the patient, who doesn't understand at all and who thinks it's all scary and strange. (Patient 2, FG2)

Communication and information exchange between GPs and specialists was mentioned in all focus groups as an important aspect of good collaboration. Several patients had the experience of visiting their GP after they had had an appointment with a hospital specialist, only to learn that the GP had not yet received information from the specialist. Information received by GPs was often incomplete, with aspects missing that patients considered important, such as complications that had occurred during their hospital stay. They also felt that information was insufficient for the GP to provide the care and explanations the patient needed.

I think that it's very important to communicate in clear language. As a specialist, you must put yourself in the patient's position, and in the GP's position. The GP visited me but had not been given enough information. Not only medical terms of what is wrong should be communicated, but also what this means for the patient and possibly for the GP and what's next, even if this isn't clear yet. (Patient 4, FG 5)

Letters in both directions often lacked information about the patient's personal context. Patients also noticed referral letters from the GP that had important medical history missing.

\section{Transparent collaborating}

Participants in the focus groups often made assumptions about collaboration because they were not involved or present when collaboration took place:

'We don't know anything about the connection between a GP and a specialist, actually... That passes us by.' (Patient 1, FG2)

Whether collaboration takes place and how well doctors work together was deduced from the patients' appointments and contacts with their doctors and the actions their doctors undertook. If procedures and follow-up appointments went smoothly, patients felt that collaboration was good, and if they noticed discrepancies between doctors or flaws in the information exchange, patients felt that collaboration was bad.

\section{Make collaboration visible}

At all moments of the patient's journey (figure 1), collaboration could be made more visible. Patients mentioned that it would help if they were able to follow the information exchange between the GP and the specialist, for example, if they received copies of letters either in print or in a digital patient system, or if telephone consultations took place in the patient's presence. To be able to understand written communication, letters should be comprehensible for patients.

Several patients mentioned that the GP and the specialist contradicted each other without explaining or considering their counterpart's perspective. Some doctors showed a disrespectful attitude towards their collaborative partner, as shown in the following quotation:

For example, the blood pressure and saturation values were all thrown away. Maybe that's not so important for the cardiologist, I don't know. But I found it, I found it strange... The specialist didn't even look at the referral letter, but away with it, immediately! Yes, I think they [specialists] feel a bit superior, if I may say so, a cut above the GP. (Patient 1, FG2)

Doctors acting in this manner gave patients a sense of bad collaboration. They found it hard to decide who they should trust, and it impaired their decision-making. Patients felt that when they were hospitalised or under intensive treatment in secondary care, their GP was not considered to be part of the treatment team, whereas to them, their GP was someone who knew them and someone they visited between hospital visits.

On the ward I was admitted to, specialists had to discover what was wrong with me, with one doctor thinking this and another thinking that. They should reach consensus about the problem and appropriate treatment together. And yes, it would be nice if they involved the GP in this process. (Patient 2, FG 1) 


\section{Clarity about roles and responsibilities}

It was often unclear to patients who they should contact in what case, or who was responsible for what part of a diagnostic trajectory.

Who is responsible for what? GPs and specialists should make arrangements about referrals. Like, if I (GP) refer the patient for this, I have to say this and that to the patient, and the specialist will take care of this and that. (Patient 3, FG3)

In some cases, patients felt that their GP had a coordinating role, and in other cases they valued contacts with a specialised nurse who was easily accessible in secondary care. Overall, they liked to have a coordinating person whom they could always contact for questions or information.

\section{Reflective practising}

In all focus groups, patients mentioned that doctors are human beings as well, and that they are allowed not to have all the answers or to make mistakes. Patients valued their doctors for acting as a human being and emphasised the importance of feedback, reflection and a learning attitude.

\section{Acknowledging mistakes and giving and receiving feedback}

Participants felt that giving and receiving feedback to each other was an important aspect of collaboration between doctors. It was their experience, however, that this did not happen very often, particularly feedback from the GP to the specialist.

And the specialist does not hear this story, what I've told the GP, but that would be good, that the specialist gets feedback...that the specialist hears what he or she has caused. (Patient 3, FG1)

One participant mentioned a very positive experience of a specialist's change of behaviour after receiving feedback from the GP.

I had a telephone appointment with my specialist because I was starting new medication, but the specialist never called. I went to the GP because I didn't feel well, and the GP called the specialist immediately... The funny thing was that after the GP had called, the specialist never forgot to call me anymore. So, it helped, this feedback. (Patient 3, FG 4)

\section{Lifelong learning}

Participants indicated that doctors should collaborate to stay up-to-date. Patients mentioned this most often about GPs and felt that specialists had to decide what should be known and learnt, which might be not only about diseases in general, but also about patient-specific, individualised protocols. Some patients mentioned that specialists could learn some lessons on communication from GPs. Both could learn from each other how to collaborate and communicate with each other.

\section{Barriers, facilitators and strategies}

Patients saw time and money constraints as the most important barriers to good collaboration. Collaboration was facilitated when the GP and specialist knew each other, when they maintained contact with their counterpart's work context, as when GPs conducted research at a university hospital, for example, or when they had gained experience in their counterpart's work context.

Strategies named to improve collaboration included a shared medical system to prevent information-exchange problems, staying in touch with their counterpart's work context and taking courses together. Another potential strategy was to visit each other's workplace.

\section{DISCUSSION}

This study enhances our understanding of the perspective on collaborative competencies of the most important stakeholder in collaborative care: the patient. This topic has hardly been studied at all. Our research design gave us the opportunity to put our question to patients personally and directly. The interaction between participants and the possibility for them to ask explanatory questions was very important, as most patients started with mentioning that collaboration was something they were unaware of. Sharing their experiences with each other helped them to reflect on the collaborative process and on the role of their doctors in it.

Results show three groups of competencies for good collaboration between doctors in primary and secondary care: relationship building, both with patients and with other doctors, transparent collaborating and reflective practising. Although we asked participants about collaboration between doctors, all focus groups mentioned doctors' relationship with patients and the importance for them to be seen as individuals and to be listened to as a proxy of collaboration between doctors. Participants mentioned that collaboration was often a black box to them, something they were unaware of and not involved in, and that its occurrence and its quality were deduced from their appointments and contacts with their doctors and the actions they undertook.

Research that includes patients is often performed in a specific patient group, such as those with a specific condition, or focuses on a specific step of the care process, such as discharge or referral. As we did not focus on a particular patient group, we provided a more holistic view of the patient perspective. We realise that our study involved only a limited number of patients and, moreover, a selected group of patients, who were willing to share their experiences and opinions about collaboration; all patients lived in the Netherlands and most came from the same region. This limits transferability. Though the groups were smaller than we had planned, we noticed that this offered more space and time for patients to share their experiences, which was necessary to address our key question. Furthermore, the groups were not as heterogeneous as we had wanted them to be, which may 
have affected the results and could also affect transferability. Although we focused on the patients' perspective, they were not involved in the design and analysis of the study. Including patients in the research team could have provided useful and different insights. Most of the researchers, finally, have a background in medical practice, which helped to understand the context but might have influenced analysis.

In a review of patient experiences of transitions, patients identified areas for improvement in four domains. ${ }^{10}$ In the domain of relationships and personal value, they noticed tensions in the relationship between doctors in primary and secondary care, which affected their experience of collaboration between doctors, as it did in our study. Based on our research, we feel that the transparency of collaborative processes and the acknowledgement of different partners in collaboration with different roles and responsibilities might help to decrease tensions between doctors as experienced by patients. Relations and communication with patients, another domain in the review, are one of the main ways in which patients can experience collaboration, and this was also confirmed in our focus groups.

In a literature review of collaborative competencies for doctors at the primary-secondary care interface, six groups of competencies were identified: (1) patient centredness: a common concern, (2) communication between doctors, (3) roles and responsibilities, (4) mutual understanding and knowledge, (5) collaborative attitude and (6) leadership (Janssen M. et al..$^{23}$ Competencies to promote collaboration between primary and secondary care doctors: An integrative review). Elements of the first five groups were also found in this focus group study. In addition, patients mentioned the importance of the relationship with the patient and transparency in the collaborative process towards patients. While the findings of the review were mainly from the doctors' viewpoint, our study from the patients' perspective showed that patients notice different aspects which could easily be adopted by doctors to improve collaboration.

Given the changes taking place in healthcare, primarysecondary care collaboration is vital to keep providing high-quality, personalised care. ${ }^{2}$ At all stages of medical education, time should be reserved for the acquisition and maintenance of those collaborative competencies that are necessary for such primary-secondary care collaboration. Using patients' experiences with the collaborative process can be used as a strong motivator. ${ }^{24}$ Patients are willing to play a role in collaboration, but to be able to do so they need to have a grasp of the process of collaboration and the people involved. At all moments of the patient's journey through primary and secondary care, therefore, collaboration between doctors could be made more visible.

If doctors are more aware of the patient journey, including the other doctors this involves and how they communicate with their patients, this can help to improve collaboration from the patient's perspective.
Doctors should realise that their way of communicating and their attitude towards their collaborative partners is sending a message to patients. Patients valued doctors for being open to feedback and for acting as 'human beings', with flaws and limitations. Reflection is now part of the professional competence of healthcare professionals. ${ }^{25}$ Learning to be a transparent and reflective doctor towards other doctors and towards patients, however, requires the support of the work environment. Reflection and feedback are obstructed by hierarchy and culture, problems in lines of communication, lack of time and increasing demands from society. ${ }^{26}$

Although we gathered new information on the patients' perspective on competencies needed for primary and secondary care doctors to collaborate smoothly, some questions have remained unanswered. How can we make sure that (future) doctors acquire these competencies? And how do we create a work environment in which these competencies, once acquired, can be developed and promoted with the goal of optimising patient-centred care? Future research should focus on answering these questions.

\section{CONCLUSION}

This focus group study enhances our understanding of the patient perspective on doctors' collaborative competencies. With this information, doctors can improve their collaborative knowledge, skills and attitudes to a level that would meet the patients' needs. Furthermore, this information can be used in the development of medical training content aiming to improve primary-secondary care collaboration. In order to provide good collaboration, doctors should build relationships, act as reflective practitioners, include patients in the collaborative process and make collaboration more explicit. All this could help to improve collaboration between primary and secondary care.

Acknowledgements The authors would like to thank all participating patients for their time and willingness to share their personal experiences and ideas about collaboration between doctors working in primary and secondary care. They thank the recruiting GPs for asking their patients to participate. They would also like to thank Tamara van Woezik for her assistance in the fifth focus group. Last, they thank Rikkert Stuve for his advice on written English language.

Contributors MJ, MHS, NDS-dH and JdG designed the research protocol. MJ guided the focus groups and was one of the coding researchers. LHJK and CRMGF assisted during the focus groups and were also involved in the coding process. All authors participated in the research discussions in which findings were discussed and themes and subthemes were abstracted. All authors were involved in several stages of manuscript drafting and revision. They all read and approved the final manuscript.

Funding The first author received funding from an educational research fund from the Radboud University Medical Center.

Disclaimer The funder had no role in the design of the study, collection and analysis of data or writing of the results.

Competing interests None declared.

Patient consent for publication Not required.

Ethics approval This study was approved by the Ethical Review Board of the Dutch Association for Medical Education (nr 620). 
Provenance and peer review Not commissioned; externally peer reviewed.

Data availability statement Data are available upon reasonable request. The datasets generated and analysed during the current study are not publicly available due to privacy concerns for the participants. They are available from the corresponding author on reasonable request.

Open access This is an open access article distributed in accordance with the Creative Commons Attribution Non Commercial (CC BY-NC 4.0) license, which permits others to distribute, remix, adapt, build upon this work non-commercially, and license their derivative works on different terms, provided the original work is properly cited, appropriate credit is given, any changes made indicated, and the use is non-commercial. See: http://creativecommons.org/licenses/by-nc/4.0/.

ORCID iD

Marijn Janssen http://orcid.org/0000-0003-4188-8889

\section{REFERENCES}

1 Barnett K, Mercer SW, Norbury M, et al. Epidemiology of multimorbidity and implications for health care, research, and medical education: a cross-sectional study. Lancet 2012;380:37-43.

2 Frenk J. Reinventing primary health care: the need for systems integration. Lancet 2009;374:170-3.

3 Wolff JL, Starfield B, Anderson G. Prevalence, expenditures, and complications of multiple chronic conditions in the elderly. Arch Intern Med 2002;162:2269-76.

4 Mastellos N, Car J, Majeed A, et al. Using information to deliver safer care: a mixed-methods study exploring general practitioners' information needs in North West London primary care. J Innov Health Inform 2015;22:207-13

5 Osborn R, Moulds D, Schneider EC, et al. Primary care physicians in ten countries report challenges caring for patients with complex health needs. Health Aff 2015;34:2104-12.

6 Haggerty JL, Reid RJ, Freeman GK, et al. Continuity of care: a multidisciplinary review. BMJ 2003;327:1219-21.

7 Preston C, Cheater F, Baker R, et al. Left in limbo: patients' views on care across the primary/secondary interface. Qual Health Care 1999;8:16-21.

8 Moore C, Wisnivesky J, Williams S, et al. Medical errors related to discontinuity of care from an inpatient to an outpatient setting. J Gen Intern Med 2003;18:646-51.

9 San Martín-Rodríguez L, Beaulieu M-D, D'Amour D, et al. The determinants of successful collaboration: a review of theoretical and empirical studies. J Interprof Care 2005;19 Suppl 1:132-47.
10 Sampson R, Cooper J, Barbour R, et al. Patients' perspectives on the medical primary-secondary care interface: systematic review and synthesis of qualitative research. BMJ Open 2015;5:e008708.

11 Beaulieu MD, Samson L, Rocher G, et al. Investigating the barriers to teaching family physicians' and specialists' collaboration in the training environment: a qualitative study. BMC Med Educ 2009:9-31.

12 Sampson R, Barbour R, Wilson P. The relationship between GPs and hospital consultants and the implications for patient care: a qualitative study. BMC Fam Pract 2016;17:45.

13 Manca D, Varnhagen S, Brett-MacLean P, et al. Respect from specialists: concerns of family physicians. Can Fam Physician 2008;54:35.e1-5:1434-5.

14 Manca DP, Varnhagen S, Brett-MacLean P, et al. Rewards and challenges of family practice: web-based survey using the Delphi method. Can Fam Physician 2007;53:278-86.

15 Stalmeijer RE, Mcnaughton N, Van Mook WNKA. Using focus groups in medical education research: AMEE guide No. 91. Med Teach 2014;36:923-39.

16 UNESCO. International standard classification of education. Available: http://uis.unesco.org/sites/default/files/documents/ international-standard-classification-of-education-isced-2011-en.pdf [Accessed 18 May 2018].

17 National AYA 'Young \& Cancer' Care Network. AYA 'Jong \& Kanker'. Available: https://ayazorgnetwerk.nl/

18 Birt L, Scott S, Cavers D, et al. Member checking: a tool to enhance Trustworthiness or merely a NOD to validation? Qual Health Res 2016;26:1802-11.

19 Braun V, Clarke V. Using thematic analysis in psychology. Qual Res Psychol 2006;3:77-101.

20 Tavakol M, Sandars J. Quantitative and qualitative methods in medical education research: AMEE guide no 90: Part II. Med Teach 2014;36:838-48.

21 Frambach JM, van der Vleuten CPM, Durning SJ. Am last page. quality criteria in qualitative and quantitative research. Acad Med 2013;88:552

22 Barry CA, Britten N, Barber N, et al. Using reflexivity to optimize teamwork in qualitative research. Qual Health Res 1999;9:26-44.

23 Janssen M, Sagasser MH, Fluit CRMG, et al. Competencies to promote collaboration between primary and secondary care doctors: an integrative review, In press.

24 Griffin A, Knight L, McKeown A, et al. A postgraduate curriculum for integrated care: a qualitative exploration of trainee paediatricians and general practitioners' experiences. BMC Med Educ 2019;19:8.

25 Epstein RM. Mindful practice. JAMA 1999;282:833-9.

26 Mann K, Gordon J, MacLeod A. Reflection and reflective practice in health professions education: a systematic review. Adv Health Sci Educ Theory Pract 2009;14:595-621. 